\title{
Manifestation of the electromagnetic proximity effect in superconductor-ferromagnet thin film structures
}

\author{
M. G. Flokstra ${ }^{1 *}$, R. Stewart ${ }^{1}$, N. Satchell ${ }^{2,3}$, G. Burnell ${ }^{3}$, H. Luetkens ${ }^{4}$, \\ T. Prokscha ${ }^{4}$, A. Suter ${ }^{4}$, E. Morenzoni ${ }^{4}$, S. Langridge ${ }^{2}$ and S. L. Lee ${ }^{1}$ \\ ${ }^{1}$ School of Physics and Astronomy, SUPA, University of St.Andrews, \\ St.Andrews KY16 9SS, United Kingdom. ${ }^{2} I S I S$, \\ Rutherford Appleton Laboratory, Oxfordshire OX11 OQX, \\ United Kingdom. ${ }^{3}$ School of Physics and Astronomy, University of Leeds, \\ Leeds LS2 9JT, United Kingdom. ${ }^{4}$ Labor für Myonspinspektroskopie, \\ Paul Scherrer Institut, CH-5232 Villigen PSI, Switzerland.
}

(Dated: August 4, 2019)

\begin{abstract}
Using the newly emerged theory model of an electromagnetic proximity effect, we demonstrate it provides a good description of our previously reported anomalous Meissner screening observed in thin film superconductor-ferromagnet proximity structures. Using the low energy muon spin rotation measurement technique we further investigate this new theory by probing directly the flux screening in various superconductor-ferromagnet proximity structures. We examine its main characteristics and find in general good agreement between theory and experiment. Understanding and control of this new proximity effect is an important step forward towards a new generation of superconducting spintronic devices.
\end{abstract}

In the field of conventional superconductor $(S)$ - ferromagnet $(F)$ proximity systems, the characteristic particle is the odd-frequency Cooper pair[1-3]. It emerges as a result of the competition between the superconducting order parameter $\Delta$ and the ferromagnetic exchange energy $E_{\text {ex }}$. The superconducting order is built from specially paired electrons (so called Cooper pairs) and they carry the superconducting properties. In a conventional superconductor, the Cooper pairs are in a singlet spin, $s$-wave orbital state, such that the total wavefunction is anti-symmetric under exchange of particles. However, the ferromagnetic order favors a parallel alignment of the electron spins and is thus destructive to the superconducting order.

This picture changes for carefully constructed thin film $S / F$ interfaces, where odd-frequency Cooper pairs can emerge. These new pairs (created from the conventional pairs) are in a triplet spin state while maintaining their $s$ wave orbital state and must thus be of odd-frequency (i.e. anti-symmetric in time) in order for the total wavefunction to be anti-symmetric under particle exchange. The two equal-spin configurations of the triplet $\left(m_{s}= \pm 1\right)$ are robust against the otherwise hostile ferromagnetic environment even in the presence of diffusive scatterers. Since they also carry a net $\operatorname{spin}(s=1)$, these equal-spin pairs pave the way for merging the fields of superconductivity with spintronics $[4,5]$ (i.e. electronics where the spin of the electron is the information carrier rather than its charge). Their odd-frequency nature is not just an abstract convenience to make the particles obey Fermi-Dirac statistics, but has very intriguing consequences. For example, it makes the pairs respond in an opposite manner to an applied magnetic field. While a

\footnotetext{
*Corresponding author. Email: mgf@st-andrews.ac.uk
}

conventional superconductor has a diamagnetic response to an applied field, for the odd-frequency pairs, a paramagnetic response is predicted $[6,7]$ which adds flux rather than expels it.

The interplay between $\Delta$ and $E_{\text {ex }}$ has been extensively studied over the past few decades and has lead to several important (experimentally observed) advances, most notably the existence, generation and control of the equalspin pairs leading to long range supercurrents[8-15] (i.e. supercurrents through ferromagnetic materials over distances vastly exceeding the singlet coherence length) and the observation of a paramagnetic Meissner response due to the presence of odd-frequency pairs[16]. Both are examples of (standard) $S / F$ proximity effects; the behavior of the superconducting pairs outside of the superconductor. Contrary to this is the inverse (or magnetic) proximity effect which predicts a spin polarization to appear inside the superconductor[17]. Observing this effect experimentally has proven difficult and while there is some evidence[18, 19], detailed measurements probing the interface region specifically have shown very different behavior[20, 21]. Very recently, new theoretical developments may finally have lifted these apparent discrepancies via something that can be called an electromagnetic (EM) proximity effect[22-25]. In essence it is the screening response of the superconductor to a vector potential at (or near) the $S / F$ interface.

In a previous paper[26] we have reported an anomalous screening behavior in a $\mathrm{Cu} / \mathrm{Nb} / \mathrm{Co}$ thin film. We observed a much enhanced screening in the trilayer compared to its $\mathrm{Nb}$ and $\mathrm{Cu} / \mathrm{Nb}$ counterpart (control) samples, contradicting theory that predicts a reduced screening due to the ferromagnet strongly suppressing superconductivity near the $S / F$ interface. For the control samples the screening could be well described within the standard London theory for thin films and also by theoretical modeling using the quasiclassical Green's func- 
tions in the Usadel framework. At the time of publication the new EM theory was not yet published and in light of this we will start by reanalyzing the $\mathrm{Cu} / \mathrm{Nb} / \mathrm{Co}$ sample within this new model. We then present experimental results investigating the manifestation of the EM proximity model in various $\mathrm{S} / \mathrm{F}$ hybrid structures and find in general good agreement between theory and experiment.

Our samples were prepared by dc magnetron sputtering on $\mathrm{Si}(100)$ substrates in a system with a base pressure of $10^{-8}$ mbar at an ambient temperature. Growth of all layers was performed at a typical Ar flow of 24 sccm and pressure of 2-3 $\mu$ bar with a typical growth rate of $0.2 \mathrm{~nm} \mathrm{~s}^{-1}$. Growth rates for each material were calibrated by fits to Kiessig fringes obtained by low angle $\mathrm{X}$-ray reflectivity measurements on single material layers. For all samples, we used $\mathrm{Nb}$ as a superconductor and we have used two different $\mathrm{Nb}$ target purities (99.99\% and 99.999\%). Our sputtered $\mathrm{Nb}$ films have a typical superconducting transition temperature $\left(T_{c}\right)$ of $8.7 \mathrm{~K}$ and a superconducting (Ginzburg-Landau) coherence length $\left(\xi_{S}\right)$ of about $10 \mathrm{~nm}$ when using the lower purity target and $11.1 \mathrm{~nm}$ when using the higher purity target. These values were determined from critical field measurements with field perpendicular to the sample plane. For all our samples, the $\mathrm{Nb}$ layers are grown with a thickness of $50 \mathrm{~nm}$ and $\mathrm{Cu}$ layers with a thickness of $40 \mathrm{~nm}$. The main effect of the different target purities is that $\mathrm{Nb}$ grown with the higher purity target has a longer mean free path and subsequently a shorter London penetration depth (about $160 \mathrm{~nm}$ ) compared to the $\mathrm{Nb}$ grown with the lower purity target which has a London pentration depth of about $270 \mathrm{~nm}[26]$.

To measure directly the local magnetic flux density inside our samples we use the low-energy muon-spin rotation (LE $\mu \mathrm{SR})$ technique[27], which has proven to be very successful in studying novel effects in $S / F$ proximity systems. The technique uses the positive muon as a local magnetic probe where the stopping depth of muons into the sample is determined by the muon energy and the depth can be tuned from about 10 to $100 \mathrm{~nm}$. This stopping depth (or stopping profile) can be calculated by a well-proven Monte-Carlo simulation[28, 29]. All LE$\mu \mathrm{SR}$ measurements were performed on the $\mu \mathrm{E} 4$ beamline at the Paul Scherrer Institut[30] in the transverse field geometry (applied field orthogonal to the muon spin direction) with the applied field direction in the plane of the sample. The lateral sizes of our samples are about $2 \times 2 \mathrm{~cm}^{2}$ such that we capture the full muon beam, which is roughly $2 \mathrm{~cm}$ in diameter. The measurement field was usually set to 300 Oe (the highest possible at the beamline). From the measurement data taken at a particular muon energy $E$ (and thus a particular probing depth profile) one can determine the average flux density $\langle B\rangle(E)$, which can also be presented as $\langle B\rangle(\langle x\rangle)$ with $\langle x\rangle$ the average probing depth of the muons at energy $E$. Using this conventional way of treating the raw detector data in combination with a series of measurements at varying implantation energy gives a reasonable approximation of the actual flux profile $B(x)$. However, in cases where the shape of the flux profile is known (either as an analytical expression or numerically determined) one can treat the measurement data imposing this profile. For example, for a superconducting film the theoretical profile is a Meissner profile and by applying this to the measurement data the magnetic field penetration depth is obtained. For our presented muon data we will show the obtained best fit for the appropriately chosen model function for $B(x)$ and also the obtained averages $\langle B\rangle(\langle x\rangle)$.

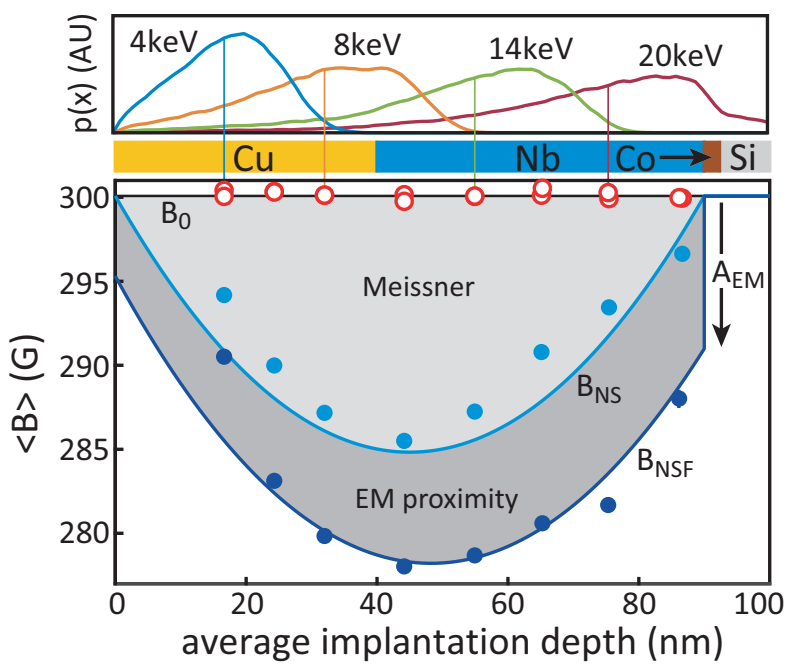

FIG. 1: Top panel: Muon stopping profiles for the $\mathrm{Cu} / \mathrm{Nb} / \mathrm{Co}$ trilayer for several implantation energies with their respective average implantation depth marked on the $\mathrm{x}$ axis. For $\mathrm{E}=20 \mathrm{keV}$ and above, the profiles extend into the Si substrate (not shown). Bottom panel: LE $\mu \mathrm{SR}$ results showing the flux profiles (solid lines) and averages (round symbols) obtained for a $\mathrm{Cu} / \mathrm{Nb}$ bilayer $(\mathrm{NS})$ and $\mathrm{Cu} / \mathrm{Nb} / \mathrm{Co}$ trilayer (NSF). Open (closed) symbols correspond to measurements taken at $T=10 \mathrm{~K}(2.5 \mathrm{~K})$. The data used is the same as the set II of Ref.[26].

We adopt the following strategy for the modeling of the flux profiles in our various samples. For our $\mathrm{Cu} / \mathrm{Nb}$ bilayers $\left(B_{\mathrm{NS}}\right)$, we assume a London type Meissner profile which has the form[31]:

$$
B_{\mathrm{NS}}(x)=B_{0} \cosh \left(\frac{x}{\lambda}-\frac{L}{2 \lambda}\right) \cosh \left(\frac{L}{2 \lambda}\right)^{-1}
$$

with $B_{0}$ the flux density of the applied (measurement) field, $\lambda$ the magnetic field penetration depth, $L$ the thickness of the bilayer and $x=0$ corresponding to the vacuum side of the $\mathrm{Cu}$ layer (where muons enter the sample). While in general the shape of the flux profile will depend on the ability of Cooper pairs to diffuse into the $\mathrm{Cu}$, and thus depends on the interface resistance and mean free path in the $\mathrm{Cu}$, we found that for our sputtered $\mathrm{Cu}$ the conditions are such that a near symmetric flux profile establishes throughout the bilayer (see Ref.[26]) thus justifying using the approach taken here. For the flux 
profile in our $\mathrm{Cu} / \mathrm{Nb} / \mathrm{Co}$ trilayers $\left(B_{\mathrm{NSF}}\right)$ we add to this the effect of the EM proximity effect originating at the $\mathrm{S} / \mathrm{F}$ interface and obtain:

$$
B_{\mathrm{NSF}}(x)=B_{\mathrm{NS}}(x)+A_{\mathrm{EM}} e^{((x-L) / \lambda)}
$$

with $A_{\mathrm{EM}}$ the strength of the EM proximity.

In Fig. 1 we show the results of applying the above flux profiles to the LE $\mu \mathrm{SR}$ measurement data, obtained on our $\mathrm{Cu} / \mathrm{Nb}$ and $\mathrm{Cu} / \mathrm{Nb} / \mathrm{Co}$ samples with a Co layer thickness of $2.4 \mathrm{~nm}$. The top panel shows the stopping profiles $(p(x))$ for several of the muon energies used, with vertical lines indicating their respective average probing depths. At the lowest energy of $4 \mathrm{keV}$ all muons stop inside the $\mathrm{Cu}$ layer while for increasing muon energy the muons penetrate deeper into the sample. The $p(x)$ shown are for the $\mathrm{Cu} / \mathrm{Nb} / \mathrm{Co}$ sample, but will look very similar for the $\mathrm{Cu} / \mathrm{Nb}$ sample (due to small fraction of muons reaching the right side of the $\mathrm{Nb}$ layer even at the highest energies used). The bottom panel shows the best fits obtained for $B_{\mathrm{NS}}$ and $B_{\mathrm{NSF}}$ (solid lines) as well as the averages obtained using the conventional data treating method (round symbols). The red (open symbols) and blue (closed symbols) belong to data taken at $T=10 \mathrm{~K}$ (with the $\mathrm{Nb}$ in the normal state) and $T=2.5 \mathrm{~K}$ respectively. The flux density of the applied measurement field is indicated by a thin solid black line $(\sim 300 \mathrm{G})$ and the highlighted areas show the contribution to $B(x)$ from normal Meissner screening $\left(B_{\mathrm{NS}}\right)$ and from the EM proximity part. For the bilayer sample we obtain $\lambda=139 \mathrm{~nm}$ and for the trilayer sample, using this value, we obtain $A_{\mathrm{EM}}=-9 \mathrm{G}$. It can be clearly seen that the anomalous behavior of the $\mathrm{Cu} / \mathrm{Nb} / \mathrm{Co}$ sample is in fact well described by this new EM proximity model. Only at the lowest muon implantation energy $(4 \mathrm{keV})$ does the model start to deviate from the actual average of the data. To look into this in more detail we apply the profile $B_{\mathrm{NSF}}$ to the measurement data taken at different energies $E$ independently to find the optimized value for $A_{\mathrm{EM}}$ as a function of energy. This is presented in Fig. 2.

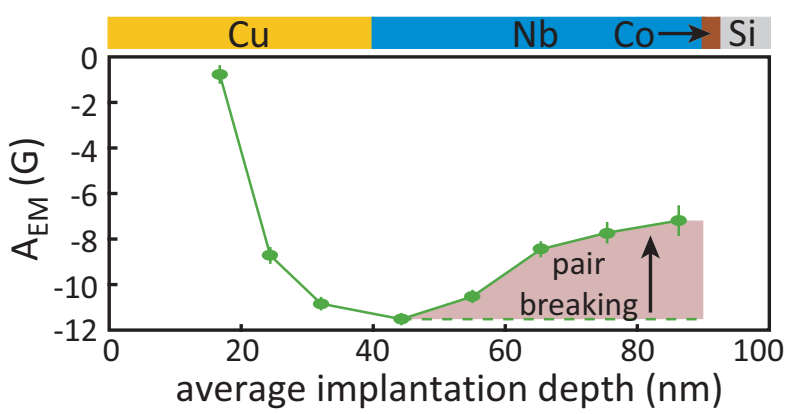

FIG. 2: LE $\mu$ SR results showing the obtained best-fit values for $A_{\mathrm{EM}}$ for the trilayer of figure 1 when applying the model function $B_{\mathrm{NSF}}$ to each measurement independently. The shaded area highlights the (possible) effect of pair breaking (see text).
For the lowest energy, indeed the data suggests that there is almost no contribution from an electromagnetic proximity effect. For all other muon implantation energies, the best fit value for $A_{\mathrm{EM}}$ are all within a small region of about $-9 \mathrm{G} \pm 2 \mathrm{G}$. The EM proximity model is thus able to give a good description of our anomalous data (apart from the small region near the surface of the sample). We note that due to pair breaking at the $\mathrm{S} / \mathrm{F}$ interface there is also an expected reduction to the screening near the $\mathrm{S} / \mathrm{F}$ interface region. In our model this would manifest as a reduction to the value of $A_{\mathrm{EM}}$ for implantation energies probing the $\mathrm{S} / \mathrm{F}$ region. We believe this to be the reason for the trend in $A_{\mathrm{EM}}$ at higher energies, as indicated by the shaded area in the figure.

The main characteristics of the EM proximity model are (1) its relatively long decay length when compared with the superconducting coherence length $\left(\lambda>>\xi_{S}\right)$, (2) the non-zero offset at the S/F interface which is strikingly different from a Meissner profile which goes to zero expulsion at the outer interfaces, and (3) its predicted dependence on the thickness of the $\mathrm{F}$ layer and direction of its magnetization. While (1) is clear from our data on the trilayer, for (2) and (3) we investigate the properties in more detail. Starting with $(2)$, we grow a $\mathrm{Cu} / \mathrm{Nb} / \mathrm{Co}$ sample but in reversed order, such that now the Co layer is on top of the $\mathrm{Nb}$ and the $\mathrm{Cu}$ on the bottom. The muons now arrive from the Co side and can probe the $\mathrm{S} / \mathrm{F}$ interface region with much more precision due to a narrower depth distribution for the low energies. By making scans as a function of energy the effective spatial resolution for these measurements is about 10-20 nm. To slow down the muons and stop them predominantly near the $\mathrm{S} / \mathrm{F}$ interface we also add a $\mathrm{Cu}$ layer on top of the $\mathrm{Co}$, such that the full layout becomes $\mathrm{Cu} / \mathrm{Co} / \mathrm{Nb} / \mathrm{Cu} / \mathrm{Si}$. Figure 3 shows the results on this inverted trilayer sample including a direct comparison with the $B_{\mathrm{NSF}}$ obtained on a regular trilayer from the same sample growth cycle (see supplementary material for more information). The circles are the $\langle B\rangle(\langle x\rangle)$ obtained for the inverted trilayer at $T=2.5 \mathrm{~K}$, while the solid line is the model function $B_{\mathrm{NSF}}$ obtained for the regular trilayer, but with inverted orientation to match the inverted sample and $B_{0}$ adjusted to the precise measurement field. To test if $B_{\mathrm{NSF}}$ is indeed a plausible solution, one can determine $\langle B\rangle(\langle x\rangle)$ from it by taking into account the stopping profiles: $\langle B\rangle(\langle x\rangle) \sim \int B(x) p(x) d x$. The result of the latter is presented by the dashed line labelled $a$ and shows a good agreement with the values actually obtained for $\langle B\rangle(\langle x\rangle)$. For comparison, the dashed line labelled $b$ is obtained when setting $A_{\mathrm{EM}}=0$ and shows a poor match with the observed values. We can thus describe the data on the inverted sample, probing in detail the $\mathrm{S} / \mathrm{F}$ interface region, without fitting the data itself but simply by extracting it from the full flux profile obtained on the regular trilayer. This demonstrates that the non-zero offset in the flux profile is indeed realistic for our muon data, but we note that due to the finite sampling width we can't rule out a fast decay of the signal into the $\mathrm{Cu}$ layer 
over a distance of $10-20 \mathrm{~nm}$.

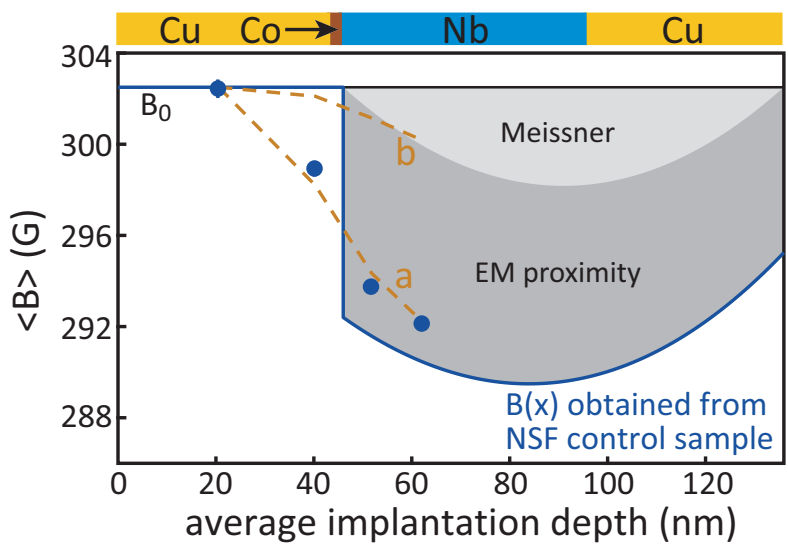

FIG. 3: LE $\mu$ SR results on the inverted trilayer (see text), with round symbols presenting the averages obtained at $T=2.5 \mathrm{~K}$ and $B_{0}$ indicating the flux of the measurement field. A direct comparison is made with $B_{\mathrm{NSF}}$ (the flux profile obtained for the regular trilayer of the same growth cycle, but mapped onto the inverted layout) by calculating $\langle B\rangle(\langle x\rangle)$ from $B_{\mathrm{NSF}}$ which is presented by the dashed line labelled $a$. The dashed line labelled $b$ is obtained when setting $A_{\mathrm{EM}}=0$.

For (3) we made a pseudo spin-valve by adding a thin Co layer to the $\mathrm{N}$ side of the $\mathrm{N} / \mathrm{S} / \mathrm{F}$ structure. By making this top $\mathrm{F}$ layer thinner compared to the bottom $\mathrm{F}$ layer ( $2 \mathrm{~nm}$ against $2.4 \mathrm{~nm}$ ), we obtain different switching fields for the two and can switch between a parallel $(\mathrm{P})$ and anti-parallel (AP) alignment between the directions of the exchange fields of the F layers. Since the theory predicts the sign of $A_{\mathrm{EM}}$ to depend on the Co thickness on a lengthscale of $\xi_{F}$ (the coherence length inside the Co, which is about $1 \mathrm{~nm}$ ), we can expect the contribution of the top Co layer to be very different from the bottom Co layer. Essentially, in the pseudo spin-valve structure we can explore the induced effects at the $\mathrm{Co} / \mathrm{Cu}(\mathrm{F} / \mathrm{N})$ interface, away from the $\mathrm{Nb} / \mathrm{Co}(\mathrm{S} / \mathrm{F})$ interface. The top panel of Fig. 4 shows the magnetic switching behavior of the pseudo spin-valve (measured at $T=50 \mathrm{~K}$ ). At an applied field of -500 Oe the Co layers are both fully saturated and aligned with the applied field ( $\mathrm{P}$ configuration). When increasing the applied field, first the thicker $(2.4 \mathrm{~nm})$ Co layer switches at about 50 Oe, setting an AP configuration, until at about 300 Oe the thinner $(2 \mathrm{~nm})$ Co layer has also fully switched resulting in a $\mathrm{P}$ configuration again. We performed $\mathrm{LE} \mu \mathrm{SR}$ measurements in the $\mathrm{P}$ configuration at +300 Oe (after saturation at +500 Oe) and in the AP configuration at +150 Oe (after saturating at $-500 \mathrm{Oe}$ ). For both, the bottom Co layer is parallel to the applied field and the top Co layer either parallel or anti-parallel to the applied field. These results are presented in the bottom panel of Fig. 4. The flux expulsion measured at $T=2.5 \mathrm{~K}$ (blue, closed symbols), compared to the normal state data measured at $T=10 \mathrm{~K}$ (red, open symbols), is significantly larger in the AP configuration compared to the $\mathrm{P}$ configuration, especially near the $\mathrm{Co} / \mathrm{Cu}$ interface where for the $\mathrm{P}$ configuration the expulsion has almost completely vanished. The result obtained for the $\mathrm{P}$ configuration is in fact very similar to results obtained on simple N/S bilayer systems (see supplementary material for more information), indicating that both EM contributions are annulling each other in this case.

Since singlets and odd-frequency triplets produce shielding contributions of opposite sign, we can use the observed Meissner magnetization to determine the dominant contribution near the $\mathrm{F} / \mathrm{N}$ interface. For our NSF trilayers, with an F layer thickness of $2.4 \mathrm{~nm}$, we measured a diamagnetic contribution from the EM proximity effect when the magnetization of the $\mathrm{F}$ layer is aligned with the applied field (see Fig.1). This implies a dominant singlet character of the Cooper pairs near the (bottom) F/S interface. In the pseudo spin-valve we observe an opposite behavior for the top $\mathrm{F}$ layer and have a smaller flux expulsion in the $\mathrm{P}$ configuration (magnetization aligned with the applied field), which becomes a larger flux expulsion when switching to the AP configuration. This allows us to conclude a dominant triplet contribution near the thinner top $\mathrm{F}$ layer. In terms of practical applications, these results show the possibility to switch between an 'ON' and 'OFF' state for a net spinpolarized supercurrent running along the $\mathrm{F} / \mathrm{N}$ interface by careful tuning of the $\mathrm{F}$ layer thicknesses.

In conclusion, we have used LE- $\mu \mathrm{SR}$ to probe directly the spatial dependence of the magnetic flux screening in $\mathrm{S} / \mathrm{F}$ thin film structures, tailored to examine the newly emerged electromagnetic proximity effect. We find in general a good agreement between this new theory and our data, which could not be explained by previous theories. Our results on the pseudo spin-valve demonstrate the possibility to control induced spin polarized supercurrents, outside of the superconductor and running along a ferromagnet - normal metal interface, which is interesting for superconducting spintronic devices.

See supplementary material for additional information about the results obtained on the $\mathrm{Cu} / \mathrm{Nb}$ and $\mathrm{Cu} / \mathrm{Nb} / \mathrm{Co}$ control samples for the inverted trilayer and the pseudo spin-valve.

We acknowledge the support of the EPSRC through Grants No. EP/I031014/1, No. EP/J01060X, No. EP/J010650/1, No. EP/J010634/1, No. EP/L015110/1, No. EP/R031924/1 and No. EP/R023522/1. This project has received funding from the European Union's Horizon 2020 research and innovation programme under the Marie Skłodowska-Curie grant agreement No. 743791 (SUPERSPIN). All muon experiments were undertaken courtesy of the Paul Scherrer Institut. 


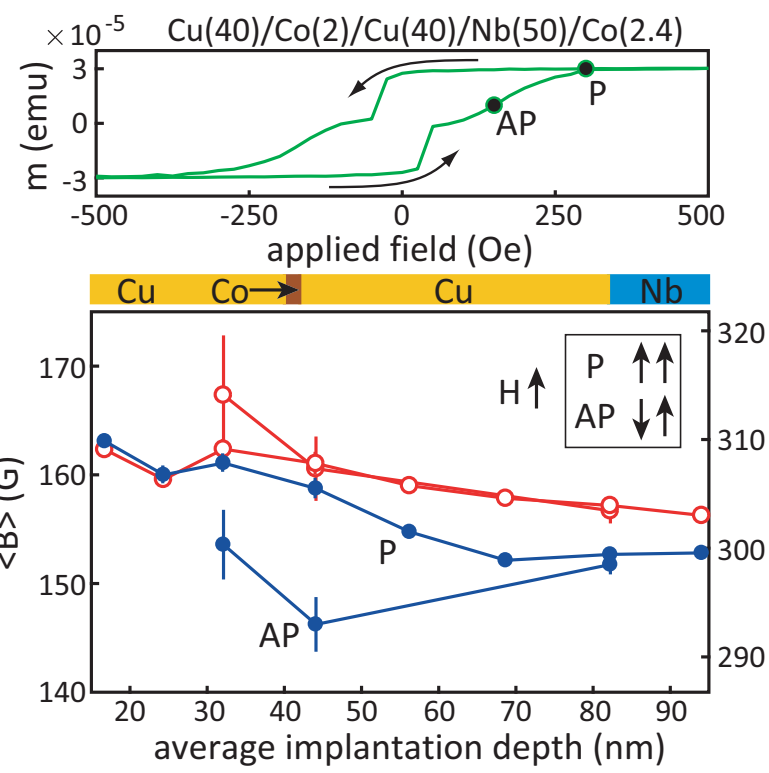

FIG. 4: Top panel: magnetization behavior of our pseudo spin-valve with numbers indicating the layer thickness in $\mathrm{nm}$. Bottom panel: LE $\mu \mathrm{SR}$ results showing the averages obtained at $T=10 \mathrm{~K}$ (red) and $T=2.5 \mathrm{~K}$ (blue) with the spin-valve in parallel (P) or anti-parallel (AP) configuration. For the P/AP configuration an applied field of about 300/150 Oe was used and data is plot against the right/left axis. The legend shows the directions of the applied field $(\mathrm{H})$ and magnetization in the top (left arrow) and bottom (right arrow) Co layer.

[1] A. I. Buzdin, Rev. Mod. Phys. 77, 935 (2005).

[2] F. S. Bergeret, A. F. Volkov, and K. B. Efetov, Rev. Mod. Phys. 77, 1321 (2005).

[3] T. Löfwander, T. Champel, J. Durst, and M. Eschrig, Phys. Rev. Lett. 95, 187003 (2005).

[4] M. Eschrig, Phys. Today 64(1), 43 (2011).

[5] J. Linder and J. W. A. Robinson, Nat. Phys. 11, 307 (2015).

[6] T. Yokoyama, Y. Tanaka, and N. Nagaosa, Phys. Rev. Lett. 106, 246601 (2011).

[7] M. Alidoust, K. Halterman, and J. Linder, Phys. Rev. B 89, $054508(2014)$

[8] R. S. Keizer, S. T. B. Goennenwein, T. M. Klapwijk, G. Miao, G. Xiao, and A. Gupta, Nature 439, 825 (2006).

[9] T. S. Khaire, M. A. Khasawneh, W. P. Pratt Jr., and N. O. Birge, Phys. Rev. Lett. 104, 137002 (2010).

[10] J. W. A. Robinson, J. D. S. Witt, and M. G. Blamire, Science 329, 59 (2010)

[11] J. W. A. Robinson, G. B. Halász, A. I. Buzdin, and M. G. Blamire, Phys. Rev. Lett. 104, 207001 (2010).

[12] J. Wang, M. Singh, M. Tian, N. Kumar, B. Liu, C. Shi, J. K. Jain, N. Samarth, T. E. Mallouk, and M. H. W. Chan, Nat. Phys. 6, 389 (2010).

[13] M. S. Anwar, F. Czeschka, M. Hesselberth, M. Porcu, and J. Aarts, Phys. Rev. B 82, 100501(R) (2010).

[14] J. Y. Gu, J. Kusnadi, and C.-Y. You, Phys. Rev. B 81,
214435 (2010).

[15] D. Sprungmann, K. Westerholt, H. Zabel, M. Weides, and H. Kohlstedt, Phys. Rev. B 82, 060505(R) (2010).

[16] A. Di Bernardo, Z. Salman, X. L. Wang, M. Amado, M. Egilmez, M. G. Flokstra, A. Suter, S. L. Lee, J. H. Zhao, T. Prokscha, E. Morenzoni, M. G. Blamire, J. Linder, and J. W. A. Robinson Phys. Rev. X 5, 041021 (2015).

[17] F. S. Bergeret, A. F. Volkov, and K. B. Efetov, Phys. Rev. B 69, 174504 (2004).

[18] R. I. Salikhov, I. A. Garifullin, N. N. Garif'yanov, L. R. Tagirov, K. Theis-Brohl, K. Westerholt, and H. Zabel, Phys. Rev. Lett. 102, 087003 (2009).

[19] J. Xia, V. Shelukhin, M. Karpovski, A. Kapitulnik, and A. Palevski, Phys. Rev. Lett. 102, 087004 (2009).

[20] M. G. Flokstra, S. J. Ray, S. J. Lister, J. Aarts, H. Luetkens, T. Prokscha, A. Suter, E. Morenzoni, and S. L. Lee, Phys. Rev. B 89, 054510 (2014).

[21] M. G. Flokstra, N. Satchell, J. Kim, G. Burnell, P. J. Curran, S. J. Bending, J. F. K. Cooper, C. J. Kinane, S. Langridge, A. Isidori, N. Pugash, M. Eschrig, H. Luetkens, A. Suter, T. Prokscha, and S. L. Lee, Nat. Phys. 12, 57 (2016).

[22] F. S. Bergeret, A. F. Volkov, and K. B. Efetov, Europhys. Lett. 66, 111 (2004).

[23] S. Mironov, A. S. Mel'nikov, and A. Buzdin, Appl. Phys. Lett. 113, 022601 (2018). 
[24] Zh. Devizorova, S. V. Mironov, A. S. Mel'nikov, and A. Buzdin, Phys. Rev. B 99, 104519 (2019).

[25] A. F. Volkov, F. S. Bergeret, and K. B. Efetov, Phys. Rev. B 99, 144506 (2019).

[26] M. G. Flokstra, R. Stewart, N. Satchell, G. Burnell, H. Luetkens, T. Prokscha, A. Suter, E. Morenzoni, S. Langridge, and S. L. Lee, Phys. Rev. Lett., 120, 247001 (2018).

[27] P. Bakule and E. Morenzoni, Contemporary Physics 45, 203 (2004).

[28] W. Eckstein, Computer Simulation of Ion-Solid Interac- tions (Springer, Berlin, Heidelberg, New York, 1991).

[29] E. Morenzoni, H. Glückler, T. Prokscha, R. Khasanov, H. Luetkens, M. Birke, E. M. Forgan, Ch. Niedermayer, and M. Pleines, Nucl. Instr. and Meth. B 192, 254-266 (2002).

[30] T. Prokscha, E. Morenzoni, K. Deiters, F. Foroughi, D. George, R. Kobler, A. Suter, and V. Vrankovic, Nucl. Instr. and Meth. A 595, 317-331 (2008).

[31] A. C. Rose-Innes and E. H. Rhoderick, Introduction to Superconductivity (Pergamon Press, 1994). 